\title{
Domain Decomposition and Additive Schwarz Techniques in the Solution of a TE Model of the Scattering by an Electrically Deep Cavity
}

\author{
Nolwenn Balin ${ }^{1,2}$, Abderrahmane Bendali ${ }^{2,3}$, and Francis Collino ${ }^{2}$ \\ 1 MBDA-France \\ ${ }^{2}$ CERFACS (http://www.cerfacs.fr/emc/balin/balin.html) \\ ${ }^{3}$ MIP
}

Summary. Two techniques are coupled to solve a model problem relative to the scattering of a 2D time-harmonic electromagnetic wave by an obstacle including an electrically deep cavity. Both of them are based on a boundary element method. The first technique uses a domain decomposition procedure to reduce the contribution of the cavity to a set of equations supported by the aperture. The second one is an additive Schwarz procedure to solve the problem after the reduction of the cavity. Numerical results are reported to give an insight into the approach.

Key words: Scattering, cavity, boundary elements, Schwarz additive method.

\section{Introduction}

It is a well-known fact for experts in stealth technology that a cavity residing in a scatterer can significantly contribute to the Radar Cross Section (RCS). Because of several difficulties, standard methods cannot be applied to solve this type of problem. Indeed, the size of the problem and the complexity of the involved phenomena (diffraction, resonance, etc.) prevent the use of available methods either direct or fast (like the fast multipole method) or asymptotic (like physical optics or geometrical theory of diffraction).

Several approaches, based on domain decomposition (DD) or hybrid methods have already been proposed: finite element-boundary integral (FE-BI) formulations (Jin [1993], Liu and Jin [2003]), multi-methods (Barka et al. [2000]) based on generalized scattering matrices, etc. However, in our opinion, none of these approaches can be considered as completely satisfactory in general. Some well-known dispersion deficiencies of FE methods can seriously damage the accuracy of the solution. Similarly, the determination of scattering matrices can rapidly become unwieldy.

We have investigated two new directions based on BI formulations to enhance the solution procedure. The first technique consists in exploiting the 
geometry of the cavity as in (Liu and Jin [2003]) to reduce its contribution to a set of equations supported only by the aperture. However, to avoid the dispersion flaws present in $\mathrm{FE}$ schemes, we use a BI formulation as well as a DD method to reduce the computing time and the memory storage. The second one is an additive overlapping Schwarz method for solving the equations on the aperture of the cavity and the rest of the boundary.

\section{Nonoverlapping Domain Decomposition Method}

\subsection{The full problem}

The geometrical data of the scattering problem are depicted in Fig. 1. They are related to a $2 \mathrm{D}$ model for the scattering of an electromagnetic wave by an open-ended thick cavity, indeed a time-harmonic $H_{z}$-wave. The scatterer is endowed with the perfect conducting boundary condition on $\Gamma$. The surrounding medium $\Omega$ is assumed to be the free-space. The unit normal to $\Gamma$ inwardly directed to $\Omega$ is denoted by $\mathbf{n}$.

Assuming an implicit time dependence in $\mathrm{e}^{-\mathrm{i} \omega t}$, we are led to solve the following boundary-value problem (see e.g., Jin [1993])

$$
\left\{\begin{array}{l}
\Delta u+k^{2} u=0 \text { in } \mathbb{R}^{2} \\
\partial_{\mathbf{n}} u=0 \text { on } \Gamma \\
\lim _{|x| \rightarrow+\infty}|x|^{1 / 2}\left(\partial_{|x|}\left(u-u^{\mathrm{inc}}\right)-\mathrm{i} k\left(u-u^{\mathrm{inc}}\right)\right)=0 .
\end{array}\right.
$$

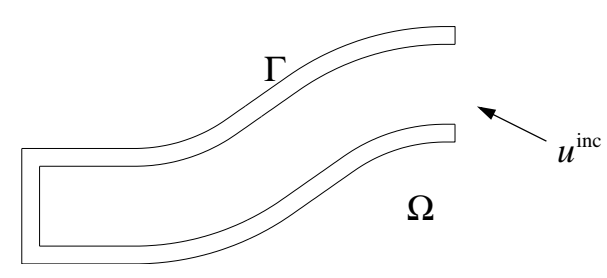

Fig. 1. The full problem.

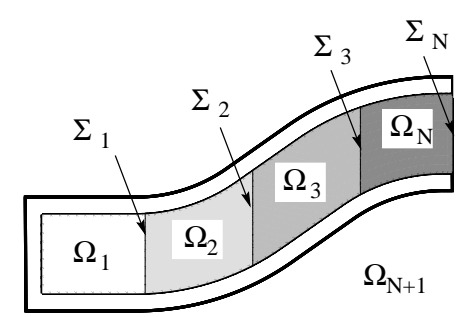

Fig. 2. The decomposition of the cavity.

\subsection{Domain decomposition and problem formulation}

The cavity is sliced into $N$ domains $\Omega_{i}(i=1, \ldots, N)$ as shown in Fig. 2. The unbounded part $\Omega_{N+1}$ of this DD of $\Omega$ lies outside the cavity. The interfaces 
$\Sigma_{i}$ between the subdomains are sectional surfaces of the cavity. Finally, the unit normal to the boundary $\partial \Omega_{i}$ of $\Omega_{i}$ outwardly directed to $\Omega_{i}$ is denoted by $\mathbf{n}_{\mathbf{i}}$.

Denoting by $u_{i}:=\left.u\right|_{\Omega_{i}}$ and by $\Gamma_{i}$ the part of $\partial \Omega_{i}$ on $\Gamma$, we are led to the following equivalent formulation of problem (1)

$$
\left\{\begin{array}{l}
\Delta u_{i}+k^{2} u_{i}=0 \text { in } \Omega_{i}, \\
\partial_{\mathbf{n}} u_{i}=0 \text { on } \Gamma_{i}, \\
\lim _{|x| \rightarrow+\infty}|x|^{1 / 2}\left(\partial_{|x|}\left(u_{N+1}-u^{\text {inc }}\right)-\mathrm{i} k\left(u_{N+1}-u^{\text {inc }}\right)\right)(x)=0,
\end{array}\right.
$$

subject to the following matching conditions

$$
u_{i}=u_{i+1} \text { and } \partial_{\mathbf{n}_{\mathbf{i}}} u_{i}+\partial_{\mathbf{n}_{\mathbf{i}+1}} u_{i+1}=0 \text { on } \Sigma_{i}, \text { for } i=1, \ldots, N .
$$

The most used BI formulations reduce the determination of $u_{i}$ in $\Omega_{i}$ to its Cauchy data $\lambda_{i}:=\left.u_{i}\right|_{\partial \Omega_{i}}$ and $p_{i}:=\left.\partial_{\mathbf{n}_{i}} u_{i}\right|_{\partial \Omega_{i}}$ (e.g. Jin [1993]). Denoting the restriction of these Cauchy data to some part of $\partial \Omega_{i}$ in an obvious way, we directly obtain the following relations from the above boundary and matching conditions

$$
\begin{gathered}
p_{i}^{\Gamma_{i}}=0, \\
\lambda_{i}^{\Sigma_{i}}=\lambda_{i+1}^{\Sigma_{i}} \text { and } p_{i}^{\Sigma_{i}}+p_{i+1}^{\Sigma_{i}}=0 .
\end{gathered}
$$

We use Rumsey's reactions principle to express the boundary and matching conditions variationally with testing functions $\lambda_{i}^{\prime}$ and $p_{i}^{\prime}$ subject to the same conditions as Cauchy data (4) and (5):

$$
\sum_{i=1}^{N+1} \int_{\partial \Omega_{i}}\left(\partial_{\mathbf{n}_{\mathbf{i}}} u_{i} \lambda_{i}^{\prime}-u_{i} p_{i}^{\prime}\right) d s=0 .
$$

Expressing $\left.u_{i}\right|_{\partial \Omega_{i}}$ and $\left.\partial_{\mathbf{n}_{\mathbf{i}}} u_{i}\right|_{\partial \Omega_{i}}$ through their integral representation in terms of $\lambda_{i}$ and $p_{i}$, we obtain the following integral equations in a straightforward way

$$
\sum_{i=1}^{N+1}\left\{\begin{array}{ll}
\lambda_{i}^{\prime T} & p_{i}^{\prime T}
\end{array}\right\} Z_{i}\left\{\begin{array}{l}
\lambda_{i} \\
p_{i}
\end{array}\right\}=\left\{\begin{array}{ll}
\lambda_{N+1}^{\prime T} & p_{N+1}^{\prime T}
\end{array}\right\} U^{\mathrm{inc}}
$$

The integrodifferential operator $Z_{i}$ becomes a complex dense matrix representing the interactions between the unknowns related to subdomain $\Omega_{i}$ once $\lambda_{i}, \lambda_{i}^{\prime}, p_{i}$ and $p_{i}^{\prime}$ have been discretized as in (Bendali and Souilah [1994]) for instance.

Clearly, this variational system has the same structure as the usual ones associated with a substructuring procedure in FE methods. It yields a linear system of the type depicted in Fig. 3. A Schur complement procedure, dealing with one subdomain at a time, can hence be used to reduce the equations relative to the cavity to a matrix coupling the Cauchy data on the aperture $\lambda_{N+1}^{\Sigma_{N}}$ and $p_{N+1}^{\Sigma_{N}}$. The procedure saves computing time and storage in a significant way. 


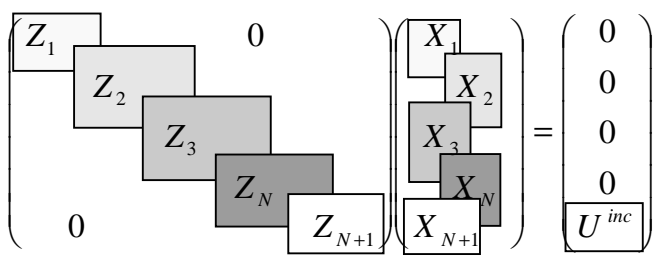

Fig. 3. Linear system

\subsection{Numerical results}

To give an insight into the performance of the method, we consider the COBRA JINA test case. To examine the effect of the exterior structure, we add a thickness (see Fig. 1) and fix the frequency at $8 \mathrm{GHz}$.

Figure 4 represents the $\mathrm{CPU}$ time necessary to compute the monostatic RCS for 361 incidences and for several decompositions of the cavity. Splitting the domain $\Omega$ into only two subdomains reduces this CPU time by a quite good factor of $65 \%$, the optimal number of subdomains being 4 for the case at hand. Meanwhile the memory storage is reduced by a factor of $60 \%$.

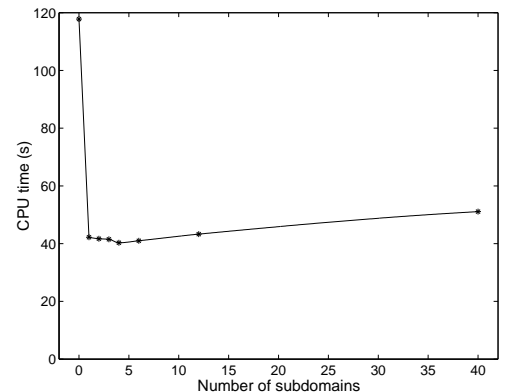

Fig. 4. Number of internal subdomains and CPU time

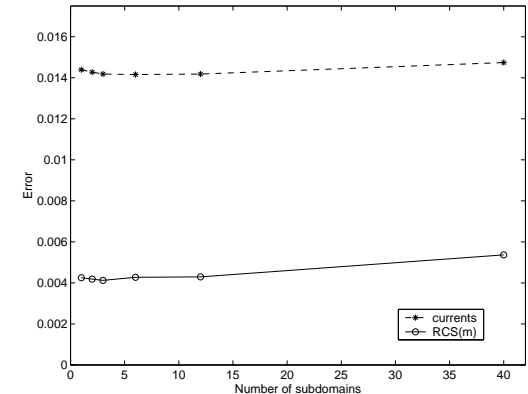

Fig. 5. Error $\left(L^{2}\right.$-norm $)$ on the currents (dashed line) and RCS (solid line)

The currents on the external boundary $\Gamma_{N+1}$ and the RCS (in m) are compared to those obtained using the direct solution (Fig. 5). Although the introduction of Cauchy data on the interfaces induces an error on the currents, they remain small and do not increase in a significant way with the number of subdomains. 


\section{Additive Overlapping Schwarz Method}

\subsection{Introduction}

The Schwarz methods (Lions [1988]) are efficient iterative processes for solving usual boundary value problems. The principle is to solve only small size problems in each subdomain in each iteration. We give an adaptation of the additive version of the Schwarz algorithm (Frommer and Szyld [1999] for example) for the problem set on the boundary of $\Omega_{N+1}$, obtained once the cavity has been reduced, to efficiently deal with its solution.

\subsection{Boundary decomposition}

We start from a generic problem like the following one

$$
X^{\prime T} B X=X^{\prime T} U
$$

assuming that this system is related to the nodal values $X$ and $X^{\prime}$ of respectively unknown and test functions defined on the boundary $\Gamma$. For the subsequent description, it will be more meaningful to denote the components of $X$ and $X^{\prime}$ as $X(x)$ and $X^{\prime}(x)$ respectively, $x$ being a node on $\Gamma$.

We consider $\Gamma_{i}(i=1, \ldots, N)$ an overlapping decomposition of $\Gamma$ (Fig. 6) as well as a partition of unity $\alpha_{i}$ associated with this covering of $\Gamma$.

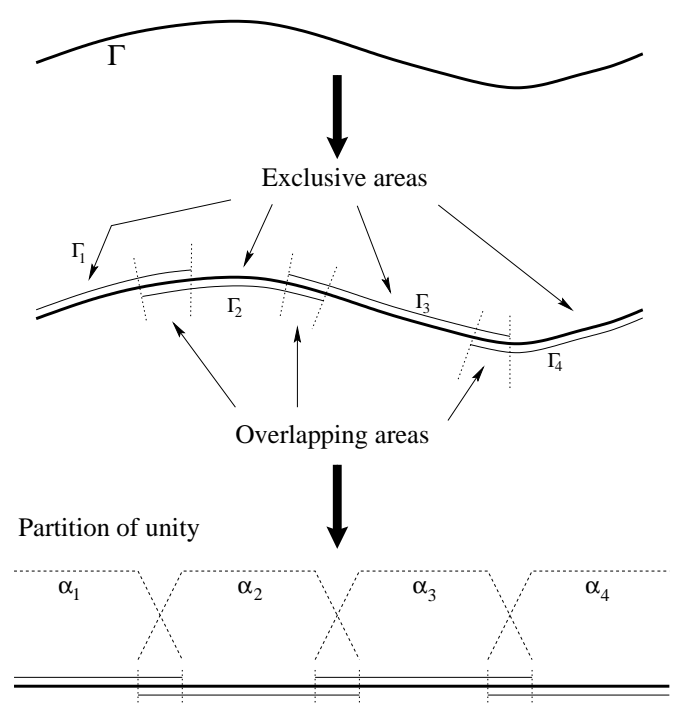

Fig. 6. Domain decomposition and partition of unity

Starting from this decomposition of the boundary, we can decompose $X$ as follows 


$$
X(x)=\sum_{j=1}^{N} \alpha_{j}(x) X_{j}(x), \quad \text { for all node } x, \quad X_{j}=\mathbb{I}_{j} X .
$$

$\mathbb{I}_{j}$ is the matrix obtained from the identity matrix $\mathbb{I}$ with the same size than $B$ by removing all the rows corresponding to a node $x$ where $\alpha_{j}(x)=0$. Now expressing the vector with the same size as $X_{j}$ and whose components are $\alpha_{j}(x) X_{j}(x)$ by means of a diagonal matrix still denoted by $\alpha_{j}$ as $\alpha_{j} X_{j}$, we can write (9) in the form of a matrix product as follows

$$
X=\sum_{j=1}^{N} \mathbb{I}_{j}^{T} \alpha_{j} X_{j}
$$

Inserting (9) in (8) and testing by $X_{i}^{\prime T} \alpha_{i} \mathbb{I}_{i}$, we are led to

$$
X_{i}^{\prime T} B_{i i} X_{i}=X_{i}^{\prime T} U_{i}-X_{i}^{\prime T} \sum_{\substack{j=1 \\ j \neq i}}^{N} B_{i j} X_{j}
$$

where

$$
B_{i j}=\alpha_{i} \mathbb{I}_{i} B \mathbb{I}_{j}^{T} \alpha_{j}, \quad U_{i}=\alpha_{i} \mathbb{I}_{i} U .
$$

We can then reconstruct $X$ to show that it solves the following fixed point problem

$$
X=\sum_{i=1}^{N} \alpha_{i} X_{i}=\sum_{i=1}^{N} \alpha_{i}\left(B_{i i}^{-1}\left(U_{i}-\sum_{j \neq i} B_{i j} X_{j}\right)\right) .
$$

This system corresponds to the classical form of the additive Schwarz algorithm (Frommer and Szyld [1999]). Once derived for a linear system $C X=$ $D$, it can be solved by the GMRES algorithm.

\subsection{Numerical results}

This method has been tested on the COBRA cavity with thin walls at a frequency of $30 \mathrm{GHz}$. This is known to be a difficult problem for the convergence of iterative methods.

\section{Distribution of the eigenvalues}

Figure 7 depicts the eigenvalues of the matrix of the initial system and those of the matrix obtained by the Schwarz procedure using a decomposition of the boundary into 75 patches. All the eigenvalues of the new matrix lie in the right half plane whereas the initial matrix has an important number of eigenvalues almost uniformly distributed in a circle centered at zero. It is wellknown that distributions of the eigenvalues of the latter type are the worst cases relatively to the convergence of iterative methods whereas the former is much more adapted to this convergence. 


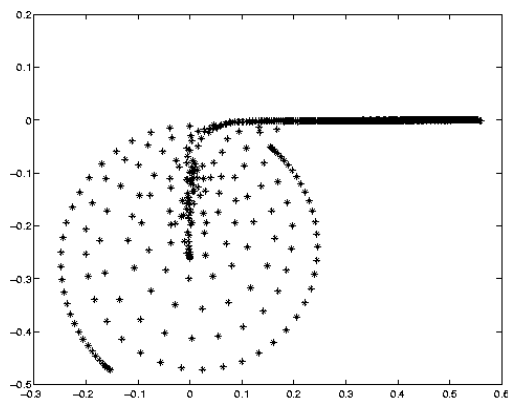

(a)

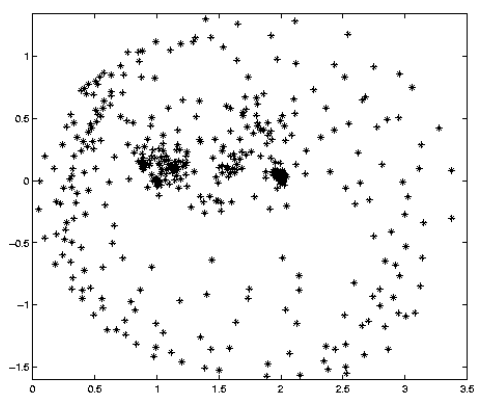

(b)

Fig. 7. Eigenvalues repartition: (a) initial matrix; (b) matrix resulting from the Schwarz method

\section{Convergence}

The results of this method have been compared to those obtained by a SParse Approximate Inverse (SPAI) preconditioning technique. The Krylov method which has been used is the GMRES algorithm with a restart every 20 iterations.

Figure 8 represents the norms of the residuals relative to the initial matrix, without any preconditioning and with a SPAI preconditioner and for the Schwarz procedure. As expected, the Schwarz technique shows a better convergence rate than the method without the preconditioner. Furthermore, the convergence rate is almost the same as that one of the SPAI method. It is worth noting that we have considered a cavity with thin walls, corresponding in fact to an open surface, which is the most unfavourable case for the convergence of the iterative process, to check its robustness.

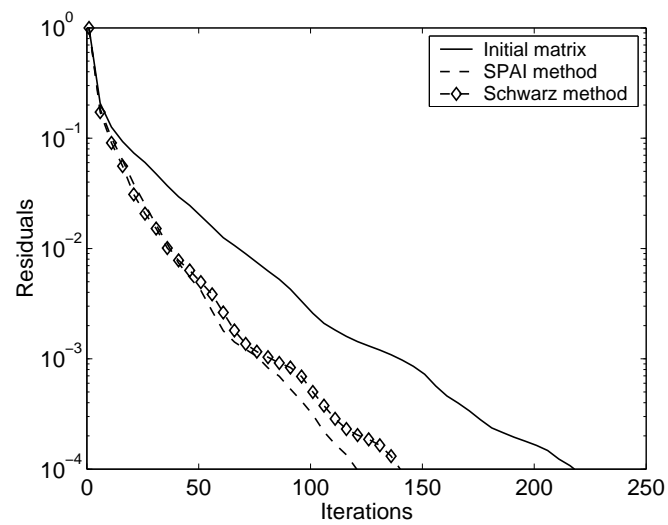

Fig. 8. Convergence rate 


\section{Conclusion and forthcoming studies}

The solution procedure proposed here has been fully validated in the $2 \mathrm{D}$ case and has efficiently handled several deep cavity problems. Work on extensions to $3 \mathrm{D}$ is currently going on. We have been inspired to do so by a prospective work in $3 \mathrm{D}$ case by M. Fares. The authors would like to acknowledge this invaluable information as well as the support of CINES which has provided the possibility in terms of massively parallel platforms to deal with such problems of really huge size.

\section{References}

André Barka, Paul Soudais, and D. Volpert. Scattering from 3D cavities with a plug\&play numerical scheme combining IE, PDE and modal techniques. IEEE Trans. Antennas and Propagation, 2000.

A. Bendali and M. Souilah. Consistency estimates for a double-layer potential and application to the numerical analysis of the boundary-element approximation of acoustic scattering by a penetrable object. Math. Comp., 62 (205):65-91, 1994.

Andreas Frommer and Daniel B. Szyld. Weighted max norms, splittings, and overlapping additive Schwarz iterations. Numer. Math., 83:259-278, 1999. URL http://www. springer-ny.com/journals/211/.

Jian-Ming Jin. The Finite Element Method in Electromagnetism. Wiley, NewYork, 1993.

Pierre-Louis Lions. On the Schwarz alternating method. I. In Roland Glowinski, Gene H. Golub, Gérard A. Meurant, and Jacques Périaux, editors, First International Symposium on Domain Decomposition Methods for Partial Differential Equations, pages 1-42, Philadelphia, PA, 1988. SIAM.

J. Liu and Jian-Ming Jin. Scattering analysis of a large body with deep cavities. IEEE Trans. Antennas and Propagation, 51(6):1157-1167, June 2003. 